\title{
A retrospective analysis of variability of clinical presentations and brain imaging findings in children with neurocysticercosis in rural population of West Bengal
}

\author{
Monojit Mondal, Biswajit Biswas ${ }^{1}$, Atanu Roy², Sumanta Laha², Rajib Das³, Abhijit Ari', Taraknath Ghosh', \\ Sabyasachi Som 1 , Mithun Konar ${ }^{2}$, Prashanth Kumar, Kanai Lal Barik', Kaustav Nayek ${ }^{4}$, Asok Kumar Datta ${ }^{5}$ \\ Resident, ${ }^{1}$ Associate Professor, ${ }^{2}$ Assistant Professor, ${ }^{3}$ RMO Cum Clinical Tutor, ${ }^{4}$ Professor, ${ }^{5}$ Professor \& Head of the Department. \\ From Department of Pediatric Medicine, Burdwan Medical College, Burdwan
}

\section{A B S T R A C T}

Background: Neurocysticercosis (NCC) is a common, widely prevalent parasitic infestation of the central nervous system in children of developing countries leading to neurological morbidities. Objectives: To study the variability of initial clinical presentations and brain imaging findings in children with NCC in the rural population of West Bengal. Methods: A retrospective analysis was done in the Department of Pediatric Medicine, Burdwan Medical College, Burdwan, India from August 2011 to August 2013. Results: Out of 952 admitted children with seizure, 113 children were diagnosed as having NCC (11.9\%). Out of them, 62 $(54.9 \%)$ were boys and $51(45.1 \%)$ were girls. The age of presentation varied from 3 years to 12 years, with mean age 9.4 years. The commonest age of presentation was between 10 to 12 years $(n=62 ; 54.9 \%)$. The maximum number of patients were from Burdwan district $(\mathrm{n}=72 ; 63.7 \%)$ followed by Birbhum $(\mathrm{n}=25 ; 22.1 \%)$. The commonest initial presentation with seizure $(n=93 ; 82.3 \%)$; partial seizure were present in 76 patients $(81.7 \%)$. On brain imaging study, the commonest location of lesions was found in the parietal region $(\mathrm{n}=69$; $61.1 \%)$, the right parietal region being more common. Single lesions were found in 102 patients(90.3\%). Conclusion: NCC is a major cause of neurological morbidity in children of developing nations and it should be suspected in any children presenting with a first episode of afebrile partial seizure. A screening brain imaging should be performed earlier to prevent undue mortality and morbidity.

KEY WORDS: Neurocysticercosis, Parasitic infestation, Central nervous system, Morbidities, Children

\section{INTRODUCTION}

NCC, an infection of the central nervous system caused by the larval stage of intestinal tapeworm Taenia solium, constitute a major public health problem for most of the developing countries like India. ${ }^{1,2}$ It has been estimated to cause at least 50,000 deaths per year. This disease is endemic in several Asian developing nations like India., ${ }^{2,3}$ The disease is transmitted to humans via the faeco-oral route when one ingests the food or water contaminated with the eggs of Taenia solium: So the disease may occur even in persons who do not eat pork. ${ }^{3,4} \mathrm{NCC}$ is more common in rural areas due to lack of hygienic practices. There is no data available on the clinical variability of presentations and brain imaging findings in children with NCC in rural population of West Bengal. This study is, therefore, conducted to evaluate these aspects of NCC in children in rural areas of this part of the globe.

\section{METHODS}

This retrospective hospital-based study was conducted in the Pediatric Medicine ward of the department of Pediatrics, Burdwan Medical College, Burdwan. Children admitted to paediatric inpatient ward from August 
2011 to August 2013 with a diagnosis of NCC on the basis of history and computed tomography (CT) scan/ magnetic resonance imaging (MRI) of the brain findings were included in this study. Patients who presented with neurological manifestation but brain imaging was not suggestive of NCC, and patients with seizures where brain imaging was not performed were excluded from the study. Data collected were analyzed using Microsoft Excel and statistical software SPSS version 21.

\section{RESULTS}

Out of 952 children admitted with seizure, 113 children were diagnosed as having NCC. Male predominance was noted with a male to female ratio of 1.2:1. The mean age of presentation was 9.4 years (ranged 3 to 12 years) with the peak age of diagnosis at 12 years. Maximum number of the patients were found in the age group 10 to 12 years $(62 ; 54.9 \%)$. The youngest age of diagnosis was at 3 years $(0.9 \%)$, while the eldest was at 12 years $(11.5 \%)$ (Table 1$)$.

The maximum number of patients were from Burdwan district ( $n=72 ; 63.7 \%)$, followed by Birbhum $(n=25$; $22.1 \%)$, Bankura $(n=8 ; 7.1 \%)$ and Murshidabad $(n=8$; 7.1\%) (Figure 1).

Our findings showed that most of the patients had mixed dietary habits. The majority of them $(n=103$; $91.2 \%)$ were non-vegetarian. Strict vegetarian were fewer in number $(n=10 ; 8.8 \%)$. The disease was found to be more prevalent amongst Hindus ( $n=99 ; 88.6 \%$ ),

\begin{tabular}{lcccc} 
Table 1: Age and sex distribution of NCC \\
\cline { 2 - 4 } & \multicolumn{3}{c}{ Group (years) } & Total \\
\cline { 2 - 4 } & $\mathbf{2 - 5}$ & $\mathbf{6 - 9}$ & $\mathbf{1 0 - 1 2}$ & \\
\hline Sex & 3 & 23 & 36 & 62 \\
Male & 1 & 24 & 26 & 51 \\
Female & 4 & 47 & 62 & 113 \\
Total & 4 & &
\end{tabular}

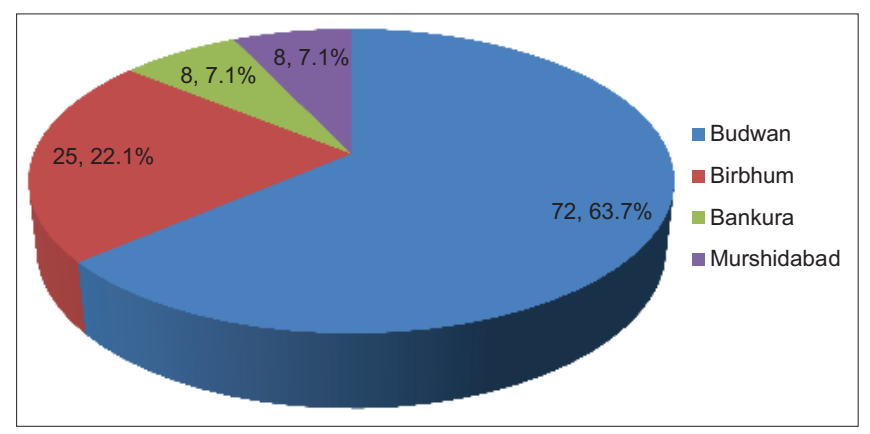

Figure 1: Geographic distribution of NCC followed by Muslims $(n=8 ; 7.1 \%)$ and Christians $(n=6$; $5.3 \%$ ) [Table 2].

The disease was found to be more common in the lower socioeconomic strata. Lower class was affected mostly $(n=54 ; 47.8 \%)$, followed by upper lower class $(n=37$; $32.7 \%)$ and then lower middle $(n=21 ; 18.6 \%)$ and upper middle $(\mathrm{n}=1 ; 0.9 \%)$.

The commonest presentation observed in the study group were seizures, noted in 93 children (82.3\%). Partial seizures were predominant $(n=76 ; 81.7 \%)$ as compared to generalized seizures $(n=17 ; 18.3 \%)$. Sudden loss of consciousness was seen in 15 patients(13.3\%), headache with or without vomiting was found in 14 patients $(12.4 \%)$, and dizziness in 3 cases $(2.7 \%)$. Besides, speech abnormalities (slurring / loss of speech) were recorded in 11 patients (9.7\%); and behavioral abnormalities in 1 $(0.9 \%)$. (Table 3$)$.

In this study, CT /MRI scan of the brain showed that $102(90.3 \%)$ patients had a single lesion, whereas multiple lesions were noticed in 11 patients $(9.7 \%)$. The approximate size of the lesions varied from $5 \mathrm{~mm}$ to $20 \mathrm{~mm}$. The most common site of involvement was parietal region, as observed in 69 patients (61.1\%). In the parietal region, lesion found on the right side in 47 cases (68.1\%), and on the left it was $22(31.9 \%)$.Frontal lesions were found in 8 cases $(16 \%)$ followed by 7 lesions $(14 \%)$ on the occipital lobe; in six cases $(12 \%)$ lesions were found in more than one lobe [Table 4].

It was seen that fifty seven $(n=57,50.4 \%)$ of the observed lesions were in a transitional stage. This was followed by

\begin{tabular}{lcccc} 
Table 2: NCC in different ethnic groups & Total \\
& \multicolumn{3}{c}{ Ethnicity } & \\
\cline { 2 - 4 } & Hindu & Muslim & Christian & \\
\hline Sex & & & 4 & 62 \\
Male & 54 & 4 & 2 & 51 \\
Female & 45 & 4 & 6 & 113 \\
Total & 99 & 8 & & \\
\hline
\end{tabular}

\begin{tabular}{lcc}
$\begin{array}{l}\text { Table 3: Signs and symptoms at presentation in } \\
\text { children with NCC }\end{array}$ & Total & Percentage \\
\hline Signs or symptoms & 93 & 82.3 \\
\hline Seizure & 76 & 81.7 \\
Partial & 17 & 18.3 \\
Generalized & 15 & 13.3 \\
Sudden loss of consciousness & 14 & 12.4 \\
Headache/vomiting & 11 & 9.7 \\
Slurring of speech/loss of speech & 3 & 2.7 \\
Dizziness & 1 & 0.9 \\
Others & & \\
\hline
\end{tabular}


calcified granuloma and active stage noted in $42(37.2 \%)$ and $14(12.4 \%)$ of the cases respectively (Table 5). Perilesional oedema was seen in $27(23.9 \%)$ cases.

Eccentric nodular hyperdensity representing the scolex was found in $11(22 \%)$ of the cases.

A lesion was said to be active if it showed a scolex with surrounding oedema or if it showed an area of hypodensity with or without surrounding oedema with a size of $<20$ $\mathrm{mm}$ in diameter. Calcified lesions $<20 \mathrm{~mm}$ in diameter were considered to be inactive lesions.

Routine investigation, which included complete blood count, liver function test, renal function test and ESR were within the normal limits. Mantoux tests were negative in all cases and size of the induration varied from 0 to $5 \mathrm{~mm}$.

\section{DISCUSSION}

This study showed that there were sixty two boys $(\mathrm{n}=62$; $54.9 \%)$ and fifty one girls $(\mathrm{n}=51 ; 45.1 \%)$ suffering from NCC. Male to female ratio was 1.2:1. Similar findings were also noted from different studies where males outnumbered females.A Study done by Singhi et al., ${ }^{5}$ found that out of 500 children with neurocysticercosis, there were 272 boys and 228 girls, with a boy to girl ratio of 1.2:1. Gauche et al., ${ }^{6}$ performed a retrospective hospital based study on 109 patients of NCC and found a ratio of male to femaleof

\begin{tabular}{lcc}
$\begin{array}{l}\text { Table 4: Brain imaging findings at presentation } \\
\text { in children with NCC }\end{array}$ & \\
\hline Lesion characteristics & Total ( $\mathbf{n = 1 1 3 )}$ & Percentage \\
\hline Number & & \\
Single & 102 & 90.3 \\
Multiple & 11 & 9.7 \\
Site of the lesion & & \\
Parietal & 69 & 61.1 \\
Right & 47 & 68.1 \\
Left & 22 & 31.9 \\
Frontal & 21 & 18.6 \\
Occipital & 10 & 8.8 \\
Temporal & 5 & 4.4 \\
Fronto parietal & 3 & 2.7 \\
Temperoparietal & 3 & 2.7 \\
Occipitoparietal & 2 & 1.8 \\
\hline
\end{tabular}

\begin{tabular}{|c|c|c|c|c|}
\hline & Active & Calcified & Transitional & Total \\
\hline \multicolumn{5}{|l|}{ Sex } \\
\hline Male & 11 & 26 & 25 & 62 \\
\hline Female & 3 & 16 & 32 & 51 \\
\hline Total & 14 & 42 & 57 & 113 \\
\hline
\end{tabular}

1.5:1. However, study done by Ruiz-Garcia, et al., ${ }^{7}$ found the female prevalence of the disease. The reason why it might be occurring more in male sex could be because they are more outgoing and are more in the habit of consuming food outside the home, especially fast food.

According to present study, the mean age of occurrence was 9.4 years (ranged 3 years to 12 years). Preschool aged children ( $<6$ years) constituting $3.5 \%$ of the total patients.A study done by Basu et al., showed that there were mean age of presentation was 10.8 years. Pozo-García et al., ${ }^{9}$ in their study in Peru, Lima showed a mean age of 7.86 years. The main reason why it is common in this age group may be because at this age the children have poor hygiene and are exposed to outside food more.

In our study, the youngest patient to suffer from NCC was a three year old girl. Our findings in terms of age groups associated with the disease are similar to those reported by Shrestha et al. ${ }^{10}$ However, one case had been reported in a patient under 2 years of age in a study conducted by Gauchan et al. ${ }^{6}$ This reflects lower incidence of NCC in younger age groups (below 3 years of age) which could be because of the prolonged incubation period of T. Solium. The reason here being that at this age the children are fed by the caretaker with food prepared at home and measures are taken to cook and feed the child with properly cooked foods.

In the present study it was found that the maximum number of patients was from Burdwan district ( $\mathrm{n}=72 ; 63.7 \%$ ), followed by Birbhum ( $\mathrm{n}=25 ; 22.1 \%)$, Bankura( $\mathrm{n}=8 ; 7.1 \%$ ) and Murshidabad( $\mathrm{n}=8 ; 7.1 \%)$ respectively. There was no study from this particular region indicating frequency of the disease occurrence. Our findings show that the majority of the patients had a mixed dietary habit.We found that 103 patients $(91.2 \%)$ were non-vegetarian whereas only 10 patients $(8.8 \%)$ were strict vegetarian.

We found that seizures were the commonest initial presentation in 93 patients $(82.3 \%)$. Among the seizure types, partial seizures were most common, accounting for $81.7 \%$ cases (partial vs generalized $77.5 \%$ Vs $22.5 \%$ ). A Study done by Singhi et al., ${ }^{5}$ showed seizures were the most common presentation accounting for $94.8 \%$ of cases; $83.7 \%$ had partial seizures. Several other studies also showed similar findings. ${ }^{11-13}$ However, in contrast, generalized seizure has been noted to be predominant in a study conducted by Gauchan et al. ${ }^{6}$ Headache and vomiting, which may be the features of raised intracranial pressure, were present in $12.4 \%$ of cases in the current study and was similar in studied conducted by Kalra et al. ${ }^{14}$ In this study no patient had fever. This similar to the study done by 
Rosenfeld et al., ${ }^{15}$ who found fever in none of the children with NCC in their study.

In this study, CT scan/MRI of the brain showed that $102(90.3 \%)$ patients had a single lesion, whereas multiple lesions were noticed in $11(9.7 \%)$ patients. Similar findings were also seen in other studies. ${ }^{6,8}$ Shrestha et al., ${ }^{11}$ in their study found single lesion in $89.8 \%$ cases. Talukdar et al., ${ }^{16}$ in their study reported similar result. In contrast, some other studies claimed that multiple lesions were predominant as compared to single lesions. ${ }^{17}$

The approximate size of the lesions varied from $5 \mathrm{~mm}$ to 20 $\mathrm{mm}$. The most common site of involvement was parietal lobe, as were observed in 69 patients $(61.1 \%)$. In the parietal region, lesions were found most commonly in the right side ( $\mathrm{n}=47 ; 68.1 \%)$. In this study, it was observed that the right parietal lobe is more frequently involved compared to left parietal lobe. Basu et al., 8 reported similar findings. However study conducted by Shrestha et al., ${ }^{11}$ revealed left parietal region involvement is predominant ( $70 \%$ vs. $30 \%$ ).

The disease activity was classified as active, transitional and inactive, based on the viability of parasites in brain imaging study. Viable cysts lack perilesional oedema, but the lesions may have an eccentric hyperdense nodule in their interior representing the scolex. The most common lesion in our study was of transitional type which was noted in 57 patients $(50.4 \%)$, whereas active lesion was the least and was recorded in only $14(12.4 \%)$ patients. Calcified lesions were found in $42(37.2 \%)$ of the cases. Cerebral oedema around the lesions was noted in $27(23.9 \%)$ of the cases. Perilesional oedema was noted mostly in a transitional stage. Only in 2 cases, calcifications were accompanied by peri-focal oedema. This localized brain oedema circumscribing ring enhancing/calcified lesions of NCC, can be considered to be the cause of seizures, which may be caused by an inflammatory response due to the liberation of antigens by the calcified cyst itself during its remodeling process. In a study by Shrestha et al., ${ }^{11}$ it was found that the lesions had mostly been in a transitional stage (61.22\%) in CT; perilesional oedema and scolex within the lesion were noted in $67.34 \%$ and $18.36 \%$ of the cases respectively. Talukdar et al., ${ }^{16}$ in their studies reported similar $\mathrm{CT}$ findings.

MRI is more sensitive in identifying the scolex within the cyst and in detection of intraventricular and subarachnoid lesion, whereas CT head is more sensitive in identifying calcified granuloma. ${ }^{18}$ Our study revealed scolex within the lesion in $11(22 \%)$ of the cases. These lesions could have been found in higher frequency if MRI head were performed in all cases. Since CT head is easily accessible and cost is very less in our institute ascompared to MRI of the brain, most children ( 99 children) had undergone CT scan of brain to diagnose NCC.

\section{CONCLUSION}

Neurocysticercosis is an important cause of seizures in children. Any child presenting with a first episode of afebrile seizure should be evaluated with an imaging of brain (CTscan/MRI); especially if they present after three years of age; provided other obvious causes of seizures are ruled out.

\section{REFERENCES}

1. Román G, Sotelo J, Del Brutto O, Flisser A, Dumas M, Wadia N, et al. A proposal to declare neurocysticercosis an international reportable disease. Bulletin of World Health Organization 2000; 78(3): 399-406.

2. Rajshekhar V, Joshi DD, Doanh NQ, van De $\mathrm{N}$ and Xiaonong Z. Taeniasoliumtaeniosis/ cysticercosis in Asia: epidemiology, impact and issues. Acta Tropica 2003; 87:53-60.

3. White AC. Neurocysticercosis: updates on epidemiology, pathogenesis, diagnosis, and management. Annu Rev Med 2000; 51:187-206.

4. Singhi $P$ and Singhi S. Neurocysticercosis in children. J Child Neurol 2004;19(7): 482-492.

5. Singhi P, Ray M, Singhi S and Khandelwal N. Clinical Spectrum Of 500 Children With Neurocysticercosis And Response Too Albendazole Therapy. J Child Neurol 2000; 15:207-213.

6. Gauchan E, Malla T, Basnet S and Rao KS. Variability of presentations and CT-scan findings in children with neurocysticercosis. Kathmandu Univ Med J (KUMJ) 2011; 9(34):17-21.

7. Ruiz-Garcia M, Gonzalez-Astiazaran A and Rueda-Franco F. Neurocysticercosis in Children: Clinical Experience in 122 Patients. Childs Nerv Syst 1997;13:608-612.

8. Basu S, Ramchandran $U$ and Thapliya A. Clinical profile and outcome of pediatric neuro-cysticercosis: A study from Western Nepal. J Pediatr Neurol 2007; 5:45-52.

9. Pozo-García MP, Campos-Olazabál P and Burneo J. Neurocysticercosis in a pediatric population in Lima: an epidemiological and clinical analysis. Rev Neurol 2003; 36 (3): 205-258.

10. Shrestha BM. Childhood Neurocysticercosis: Clinico-radiological profile \& outcome. J Nepal Paediatr Soc 2008; 28 (1): 14-16.

11. Shrestha B, Mainali $P$, Sayami $S$ and Shrestha OK. Clinicoradiological Aspects of Neurocysticercosis in Pediatric Population in a Tertiary Hospital. J Nepal Med Assoc 2013; 52 (191):483-488.

12. Carabin $H$, Ndimubanzi $P C$, Budke CM, Nguyen $H$, Qian $Y$, Cowan LD, et al. Clinical manifestations associated with neurocysticercosis: a systematic review. PLoS Negl Trop Dis 2011;5(5):e1152.

13. Patil TB and Paithankar MM. Clinico-radiological profile and treatment outcomes in neurocysticercosis: A study of 40 patients. Annals of Tropical Medicine and Public Health 2010;3(2):58.

14. Kalra $\vee$ and Sethi A. Childhood NeurocysticercosisEpidemiology, Diagnosis and Course. ActaPaediatrica Japonica 1992;37:365-370.

15. Rosenfeld EA, Byrd SE and Shulman ST. Neurocysticercosis among children in Chicago. Clin Infect Dis 1996; 23(2):262-268. 
16. Talukdar B, Saxena A, Popli VK and Choudhury V. Neurocysticercosis in children: clinical characteristics and outcome. Ann Trop Pediatr 2002; 22:333-339.

17. Joshi DD, Bista PR, Ito A and Yamasaki H. Present situation of porcine taeniasis and human cysticercosis in Nepal. Southeast Asian J Trop Med Public Health 2007;38Suppl 8:144-150.

18. Garcia $\mathrm{HH}$ and Del Brutto $\mathrm{OH}$. A Imaging findings in neurocysticercosis; ActaTropica2003; 87:71-78.

Authors Contribution:
MM, BB,AR - design of study, literature search, statistical analysis and manuscript preparation; SL,RD,AA - contributed to work design and reviewof manuscript;
TG,SS,MK,PK - data collection,editing and manuscript revision, KLB,KN, AKD - review of literature,editing, statistical analysis and overall supervision.

Source of Support: Nil, Conflict of Interest: None declared. 